Ann. Biol. anim. Bioch. Biophys., 1977, 17 (4), 615-620.

\title{
Etude in vivo chez le rat nourri du métabolisme intestinal et hépatique des acides aminés.
}

\author{
par C. DEMIGNÉ, Jocelyne AUFRERE, C. REMESY
}

Station de Physiopathologie de la Nutrition, I.N.R.A., Theix, Saint Genès Champanelle, 63110 Beaumont.

Summary. In vivo studies of aminoacid metabolism in rat intestine and liver.

Amino acid differences between the portal vein and artery and hepatic and portal veins were studied in Sherman rats fed a 13 p. 100 casein diet under Nembutal anaesthesia.

Alanine reaching the portal vein came not only from casein hydrolysis, but also from transamination of glutamic and aspartic acids in the intestinal cells and from deaminated glutamine. Aminoacid release into the portal vein was compared to overall liver balance, taking into account the flow rate of the hepatic artery. The liver uptake of alanine, glycine and aromatic amino acids was high $(78,82$ and $64-70$ p. 100, respectively), whereas that of serine and threonine was low (49 and 43 p. 100, respectively). Only a small fraction of branched-chain amino acids was taken up by the liver, the main uptake of these occuring in the peripheral tissues.

\section{Introduction.}

Le méłabolisme des acides aminés a été étudié chez le rat par diverses techniques (liaisons entre nutrition et concentrations tissulaires, bilans de digestibilité, évaluation du turnover des acides aminés, etc...). Toutefois, les techniques physiologiques par différences artérioveineuses ont été moins pratiquées en raison des difficultés de mesure des débits sanguins, indispensables pour disposer de données quantitatives. Les techniques de mesure de débit, délicates à mettre en œuvre sur le chien, le porc ou les ruminants, sont mal adaptées aux petits rongeurs. Les différences artérioveineuses sans mesure de débit ne sont pourtant pas sans intérêt, en particulier au niveau splanchnique si l'on veut comparer les acides aminés absorbés à ceux captés par le foie (Bloxam, 1972 ; Yamamoto et 1974). Le débit de la veine porte conditionnant le débit hépatique (70 p. 100 environ du débit total), le seul problème sera les fluctuations éventuelles du débit de l'artère hépatique.

Cet article a pour objeł de décrire les caractéristiques de la captation hépatique des acides aminés en période absorptive avec un régime comportant 13 p. 100 de caséine. 


\section{Matériel et méthodes.}

Des rats mâles Sherman de $200 \mathrm{~g}$ ont été nourris avec un régime apportant, en pourcentage : amidon de froment 79 , caséine 13 , huile de maïs 2 , minéraux 5 , vitamines 1. Les animaux ont été conditionnés à absorber leur nourriture en $8 \mathrm{~h}$, la phase obscure étant réglée de $9 \mathrm{~h}$ à $17 \mathrm{~h}$. Les rats ont été anesthésiés au Nembutal $(40 \mathrm{mg} / \mathrm{kg}$ s.c.) environ $4 \mathrm{~h}$ après le début de la prise de nourriture. Après laparotomie $1 \mathrm{ml}$ de sang a été prélevé soit dans la veine porte ef l'aorte, soit dans une veine sus-hépatique (à l'intérieur du lobe droit) et la veine porte. Après centrifugation, les plasmas sont congelés à $-20^{\circ} \mathrm{C}$. Les foies sont prélevés et broyés immédiatement dans $\mathrm{HClO}_{4} \quad 0,4 \mathrm{M}(7 \mathrm{v})$. Après centrifugation, le surnageant perchlorique est neutralisé à $\mathrm{pH} 7,5$ par $\mathrm{K}_{2} \mathrm{CO}_{3}$ afin d'éviter l'hydrolyse de la glutamine ef de l'asparagine et conservé à $-20^{\circ} \mathrm{C}$.

Dosage des acides aminés : les plasmas sont déprotéinisés par l'acide sulfosalicylique 4 p. $100(1 v+4 v)$ immédiatement avant l'analyse. Les échantillons hépatiques sont ramenés à pH 2,2 au moment de l'analyse. L'analyseur type LKB 4101 est équipé d'une colonne de $6 \times 350 \mathrm{~mm}$ remplie de résine Aminex A9, l'élution utilise 3 tampons lithium : $0,20 \mathrm{M}, \mathrm{pH} 2,57(60 \mathrm{mn}) ; 0,40 \mathrm{M}, \mathrm{pH} 3,00(120 \mathrm{mn})$ et 1,66 M, $\mathrm{pH} 3,60(120 \mathrm{mn})$; température $34^{\circ} \mathrm{C}$ pendant $60 \mathrm{mn}$ puis $68^{\circ} \mathrm{C}$, débit $36 \mathrm{ml} / \mathrm{h}$.

\section{Résultats et discussion.}

La prise de nourriture $(20,8 \pm 0,7 \mathrm{~g} / j$ our $)$ étant étalée sur une période limitée, l'estomac des rats est plein au moment des prélèvements et les animaux sont en pleine phase absorptive ; toutefois les fluctuations du transit et de la vidange gastrique ne sont pas à négliger. La croissance des rats était de $4 \mathrm{~g} /$ jour. La caséine est particulièrement riche en acide glutamique dont 46 p. 100 seraient sous forme de glutamine (Yamamoto et al., 1974), de même 66 p. 100 de l'acide aspartique seraient sous forme d'asparagine.

Absorption et métabolisme intestinal (tabl. 1).

En l'absence de mesures de débit, les différences artérioveineuses n'ont de signification que pour des comparaisons entre métabolites. Dans ces conditions, on constate :

Un bilan artérioveineux nul pour les acides glutamique, aspartique ef la glutamine, parallèlement à l'apparition de quantités élevées d'alanine provenant de la métabolisation de ces acides par l'intestin grêle (Parsons et Volman-Mitchell, 1974 ; Windmueller et Spaeth, 1975). Le problème de la glutamine est plus complexe : l'absorption est sans doute masquée par l'utilisation permanente de la glutamine sanguine (Aikawa ef al., 1973). L'asparagine, beaucoup moins abondante dans la caséine, semble peu métabolisée par la paroi puisque bien absorbée.

Si l'apparition dans le sang porte de quantités d'alanine disproportionnées à son taux dans la caséine s'explique facilement, il n'en est pas de même pour la glycine, 
la lysine et dans une moindre mesure la proline. Il faut noter que Nasset et Ju (1975) ont observé un enrichissement des contenus d'intestin grêle en glycine et lysine mais non en proline.

\section{TABLEAU 1}

Concentrations en acides aminés, en lactate et en glucose dans l'aorte abdominale et la veine porte chez des rats nourris. Les différences artérioveineuses (P-A) ont été également calculées pour une teneur de $1 \mathrm{mmole} / \mathrm{g}$ de caséine pour chaque acide aminé. Les résultats sont les moyennes de 3 expériences utilisant à chaque fois un pool de 7 animaux $(\bar{x} \pm \sigma / \sqrt{ } N)$.

$\begin{array}{ccccc}\begin{array}{c}\text { Caséine } \\ (\mathrm{mmoles} / \mathrm{g})\end{array} & \begin{array}{c}\text { Artère }(\mathrm{A}) \\ (\mathrm{mM})\end{array} & \begin{array}{c}\text { Veine porte }(\mathrm{P}) \\ (\mathrm{mM})\end{array} & \begin{array}{c}P-A \\ (\mathrm{mM})\end{array} & \begin{array}{c}P-A \text { pour } \\ 1 \text { mmole } / \mathrm{g} \\ \text { dans la caséine }\end{array}\end{array}$

\begin{tabular}{|c|c|c|c|c|c|}
\hline Ac. Aspartique * & 0,170 & $0,01<$ & - & - & - \\
\hline Asparagine * & 0,330 & $0,16 \pm 0,02$ & $0,26 \pm 0,03$ & $0,10 \pm 0,01$ & 0,30 \\
\hline Ac. Glutamique * & 0,792 & $0,05 \pm 0,02$ & $0,07 \pm 0,02$ & $0,02 \pm 0,01$ & 0,025 \\
\hline Glutamine * & 0,675 & $0,59 \pm 0,06$ & $0,60 \pm 0,07$ & $0,01 \pm 0,02$ & - \\
\hline Alanine $\ldots$ & 0,422 & $0,77 \pm 0,08$ & $1,28 \pm 0,05$ & $0,52 \pm 0,03$ & 1,23 \\
\hline Glycine ..... & 0,244 & $0,18 \pm 0,01$ & $0,29 \pm 0,01$ & $0,11 \pm 0,02$ & 0,45 \\
\hline Proline ...... & 0,789 & $0,58 \pm 0,02$ & $0,85 \pm 0,09$ & $0,27 \pm 0,03$ & 0,34 \\
\hline Sérine ...... & 0,478 & $0,33 \pm 0,04$ & $0,47 \pm 0,03$ & $0,14 \pm 0,02$ & 0,29 \\
\hline Thréonine & 0,322 & $0,47 \pm 0,04$ & $0,57 \pm 0,04$ & $0,10 \pm 0,02$ & 0,31 \\
\hline Valine ....... & 0,578 & $0,29 \pm 0,02$ & $0,39 \pm 0,03$ & $0,10 \pm 0,01$ & 0,17 \\
\hline Isoleucine .... & 0,389 & $0,11 \pm 0,02$ & $0,20 \pm 0,03$ & $0,09 \pm 0,01$ & 0,23 \\
\hline Leucine ...... & 0,689 & $0,18 \pm 0,02$ & $0,29 \pm 0,01$ & $0,11 \pm 0,01$ & 0,16 \\
\hline Tyrosine $\ldots \ldots \ldots$. & 0,278 & $0,10 \pm 0,02$ & $0,15 \pm 0,02$ & $0,05 \pm 0,01$ & 0,18 \\
\hline Phénylalanine .... & 0,278 & $0,08 \pm 0,01$ & $0,12 \pm 0,01$ & $0,04 \pm 0,01$ & 0,14 \\
\hline Méthionine ...... & 0,211 & $0,14 \pm 0,02$ & $0,20 \pm 0,02$ & $0,055 \pm 0,010$ & 0,26 \\
\hline Lysine $\ldots \ldots \ldots \ldots$ & 0,489 & $0,68 \pm 0,05$ & $0,85 \pm 0,04$ & $0,17 \pm 0,03$ & 0,35 \\
\hline Histidine ..... & 0,189 & $0,09 \pm 0,02$ & $0,12 \pm 0,02$ & $0,03 \pm 0,01$ & - \\
\hline Citrulline..$\ldots \ldots$ & - & $0,10 \pm 0,02$ & $0,12 \pm 0,02$ & $0,02 \pm 0,02$ & 一 \\
\hline Ornithine $\ldots \ldots \ldots$ & - & $0,06 \pm 0,01$ & $0,05 \pm 0,02$ & $-0,01 \pm 0,01$ & - \\
\hline Arginine & 0,189 & $0,10 \pm 0,01$ & $0,14 \pm 0,02$ & $0,04 \pm 0,01$ & - \\
\hline Total & & & & 1,97 & \\
\hline $\begin{array}{l}\text { Glucose } \\
\text { Lactate }\end{array}$ & & $8,20 \pm 0,20$ & $13,25 \pm 0,35$ & $5,05 \pm 0,33$ & \\
\hline & & & $3,22 \pm 0,15$ & $0,88 \pm 0,07$ & \\
\hline
\end{tabular}

* Les teneurs en glutamine et en asparagine de la caséine ont été calculées en utilisant les pourcentages établis par Yamamoto ef al. (1974).

Si on ramène les différences $\mathrm{P}-\mathrm{A}$ de la sérine, thréonine, méthionine el asparagine à une teneur identique dans la caséine on obtient des valeurs voisines, entre 0,26 et $0,31 \mathrm{mM}$. Ce même calcul, en tenant compte de l'utilisation des acides glutamique ef aspartique ainsi que de la glutamine, donne $0,25 \mathrm{mM}$ pour l'alanine. La plupart des autres acides aminés apparaissent en proportion moindre ; il est remarquable que l'ensemble des acides aminés non indispensables soił retrouvé dans la veine porte en pourcentage supérieur à celui de la caséine :ce phénomène peut provenir de transaminations affectant certains acides aminés indispensables (Ichihara, Noda ef Goto, 1975), de la dilution par les sécrétions d'azote endogène (Nasset ef Ju, 1961 ; 
Fauconneau et Michel, 1970 ; Adibi et Mercier, 1973) dont la composition peut être éloignée de celle de la caséine. L'intervention de facteurs tels que les différences d'hydrolyse ou d'absorption, et l'incorporation d'acides aminés dans les protéines intestinales ont été analysés récemment par Rérat, Corring et Laplace (1976).

Méfabolisme hépatique (tabl. 2).

Les différences P-S ne donnent pas une idée exacte des bilans hépatiques, si on néglige les différences entre veine sus-hépatique et artère. Nous avons admis que

\section{TABLEAU 2}

Concentrations en acides aminés dans la veine porte, la veine sus-hépatique et le foie de rats nourris (les différences veine porte - veine sus hépatique P-S positives correspondent à une captation). Le pourcentage de captation hépatique et celui d'utilisation hépatique par rapport à l'absorption ont été calculés sur la base débit porte $=70 \mathrm{p} .100 \mathrm{du}$ débit hépatique. Les résultats sont les moyennes de 3 expériences utilisant un pool de 7 animaux $(\bar{x} \pm \sigma / \sqrt{N})$ sauf pour le foie, dosé à partir d'un pool de 10 animaux.

\begin{tabular}{|c|c|c|c|c|c|c|}
\hline & $P$ & Foie & $S$ & P-S & $\begin{array}{l}\text { p. } 100 \text { de } \\
\text { captation } \\
\text { hépatique }\end{array}$ & $\begin{array}{c}\text { p. } 100 \\
\text { d'utilisation } \\
\text { hépatique } \\
\text { par rapport } \\
\text { à l'absorption }\end{array}$ \\
\hline 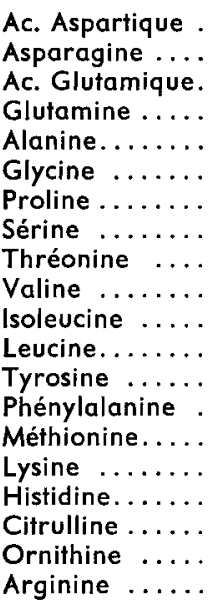 & $\begin{array}{l}0,01< \\
0,26 \pm 0,03 \\
0,07 \pm 0,02 \\
0,60 \pm 0,07 \\
1,29 \pm 0,05 \\
0,29 \pm 0,01 \\
0,85 \pm 0,09 \\
0,47 \pm 0,03 \\
0,57 \pm 0,04 \\
0,39 \pm 0,03 \\
0,20 \pm 0,03 \\
0,29 \pm 0,01 \\
0,15 \pm 0,02 \\
0,12 \pm 0,01 \\
0,20 \pm 0,02 \\
0,85 \pm 0,04 \\
0,12 \pm 0,02 \\
0,12 \pm 0,02 \\
0,05 \pm 0,02 \\
0,14 \pm 0,02\end{array}$ & $\begin{array}{l}0,74 \\
\overline{1,88} \\
3,24 \\
2,26 \\
1,66 \\
\overline{0} \\
0,57 \\
0,42 \\
0,20 \\
0,09 \\
0,17 \\
0,05 \\
0,06 \\
- \\
0,53 \\
- \\
- \\
-\end{array}$ & $\begin{array}{c}0,01< \\
0,16 \pm 0,02 \\
0,07 \pm 0,02 \\
0,70 \pm 0,10 \\
0,85 \pm 0,06 \\
0,20 \pm 0,01 \\
0,68 \pm 0,08 \\
0,38 \pm 0,03 \\
0,51 \pm 0,03 \\
0,34 \pm 0,03 \\
0,16 \pm 0,02 \\
0,23 \pm 0,02 \\
0,11 \pm 0,01 \\
0,09 \pm 0,01 \\
0,75 \pm 0,10 \\
= \\
0,12 \pm 0,01 \\
-\end{array}$ & $\begin{array}{c}0,10 \pm 0,01 \\
-0,10 \pm 0,02 \\
0,44 \pm 0,04 \\
0,09 \pm 0,01 \\
0,17 \pm 0,04 \\
0,09 \pm 0,01 \\
0,06 \pm 0,02 \\
0,05 \pm 0,02 \\
0,04 \pm 0,02 \\
0,06 \pm 0,02 \\
0,04 \pm 0.01 \\
0,03 \pm 0,01 \\
\pm \\
0,10 \pm 0,02 \\
- \\
-0,07 \pm 0,02 \\
-\end{array}$ & $\begin{array}{r}\overline{31} \\
\overline{-} \\
25 \\
25 \\
12 \\
11 \\
6 \\
6 \\
8 \\
11 \\
19 \\
17 \\
- \\
6 \\
- \\
- \\
-\end{array}$ & $\begin{array}{l}\overline{100} \\
- \\
\overline{78} \\
82 \\
47 \\
49 \\
43 \\
29 \\
21 \\
35 \\
71 \\
64 \\
- \\
41 \\
- \\
- \\
-\end{array}$ \\
\hline
\end{tabular}

le débit porte représentait 70 p. 100 du débit hépatique (Greenway et Stark, 1971). Des fluctuations de \pm 10 p. 100 autour de la valeur 30 p. 100 pour le débit de l'artère hépatique modifient assez peu le bilan de la plupart des acides aminés, sauf ceux qui présentent des différences $\mathrm{P}$-A très élevées. Sur cette base, nous avons calculé : 
- Le pourcentage de captation hépatique de chaque acide aminé $0,7(\mathrm{P}-\mathrm{S})+$ $0,3(\mathrm{~A}-\mathrm{S}) / 0,7 \mathrm{P}+0,3 \mathrm{~A}$ (par ex. : alanine $=25$ p. 100);

- Le pourcentage d'utilisation hépatique des acides aminés, par rapport à l'absorption au niveau de la veine porte : 0,7 (P-S) + 0,3 (A-S)/0,7 (P-A) (par ex. : alanine $=78$ p. 100).

II existe une complémentarité entre métabolismes intestinal et hépatique : les acides aminés les plus captés en valeur absolue sont l'alanine, la proline, la glycine, l'asparagine, la sérine ef la lysine. Les pourcentages de captation les plus élevés (25 à 30 p. 100) sont ceux de l'alanine ef de la glycine, puis ceux des acides aminés aromatiques (16 à 18 p. 100) ; ces pourcentages sont faibles pour la thréonine, la valine, l'isoleucine et la lysine.

L'utilisation hépatique des acides aminés libérés dans la veine porte est très importante : asparagine (100 p. 100), glycine ef alanine ( 80 p. 100), tyrosine ef phénylalanine (60 à 70 p. 100), proline, sérine, thréonine et lysine (40 à 50 p. 100). Ce taux ne tombe guère en dessous de 20 p. 100 pour les acides peu captés par le foie (acides aminés ramifiés). Le foie capte donc une quantité d'acides aminés sans commune mesure avec son importance pondérale (3,7 p. 100 du poids vif).

Les concentrations hépatiques en acides aminés sont très différentes de celles du plasma, d'autant plus que le foie prélevé conserve une proportion notable de sang $(0,24 \mathrm{ml} / \mathrm{g}$ selon Greenway ef Stark, 1971). Le foie est riche en acides aspartique et glutamique, en glutamine, alanine et glycine. Toutefois, ces gradients ne permettent pas de prévoir a priori le sens et l'intensité des échanges entre foie et plasma : on enregistre une faible libération de glutamine dans le sens du gradient, mais une forte pénétration de l'alanine et de la glycine contre le gradient. Par contre, alors qu'il existe un certain équilibre entre foie et plasma pour la thréonine et la sérine, les concentrations intracellulaires sont très faibles pour les autres acides aminés, sauf la lysine : les gradients sont alors en faveur de la captation, pourtant faible pour les acides aminés ramifiés. Il semble donc exister au niveau de la membrane de l'hépatocyte des phénomènes de perméabilité et de transport extrêmement sélectifs. Certaines caractéristiques de ces processus de captation ont été montrés : augmentation avec le taux protéique (Tews, Colosi ef Harper, 1975), l'AMPc ef le glucagon (Malette, Exton et Park, 1969) et le jeûne (Adibi, Morse et Mirsky, 1976).

Les acides aminés les plus abondamment libérés dans l'intestin participent de façon prépondérante à l'uréogenèse (alanine, glycine, proline ef sérine), voire à la synthèse de l'important pool intracellulaire de glutamine. Lorsque le foie reçoit un apport massif en glucose l'utilisation des chaînons carbonés des acides aminés n'est pas orientée vers la néoglucogenèse (Kaplan ef Piłot, 1970 ; Mc Daniel, 1975) mais plutôt vers la lipogenèse et l'oxydation (Peret et al., 1975).

Une grande part des acides aminés captés participe aux synthèses protéiques : protéines plasmatiques ou protéines cellulaires. Le foie constitue certainement un site privilégié dans la fabrication des protéines « labiles » lorsque l'apport en acides aminés est associé à un apport en glucides important (Munro, 1964).

En conclusion, le modèle expérimental utilisé pour l'étude détaillée du métabolisme splanchnique donne des résultats cohérents si l'on tient compte du débit de l'artère 
hépatique. En négligeant ce point, Yamamoto et al. (1974) sont parvenus à des conclusions paradoxales (utilisation par le foie de la presque totalité des acides aminés absorbés).

Commission CNERNA Digestion-Absorption, Tours, 13 novembre 1976.

\section{Références}

ADIBI S. A., MERCIER D. W., 1973. Protein digestion in human intestine as reflected in luminal, muccsal and plasma amino acid concentration after meals. J. clin. Invest., 52, 1586-1594.

ADIBI S. A., MORSE E. L., MIRSKY I. A., 1976. Dietary regulation of liver and muscle transport of amino acid. Amer. J. Physiol., 230, 245-250.

AIKAWA T., MATSUTAKA H., YAMAMOTO H., OKUDA T., ISHIKAWA E., KAWANO T., MATSUMAKA E., 1973. Gluconeogenesis and amino acid metabolism. Il. Interorganal relations and role of glutamine and alanine in the amino acids metabolism of fasted rats. J. Biochem., 74, 1003-1017.

BLOXAM D. L., 1972. Nutritional aspects of amino acid metabolism. 2. The effects of starvation on hepatic portal venous differences, plasma amino acid concentration and on liver aminoacid concentration in the rat. Br. J. Nutr., 27, 233-247.

FAUCONNEAU G., MICHEL M. C., 1970. In MUNRO H. N. Mammalian protein metabolism, vol. 4, 481, Acad. Press, N. Y., London.

GREENWAY C. V., STARK R. D., 1971. Hepatic vascular bed. Physiol. Rev., 51, 23-65.

ICHIHARA A., NODA C., GOTO M., 1975. Transamination of branched chain amino acids. High activity in stomach and pancreas. Biochem. Biophys. Res. Comm., 67, 1313-1318.

KAPLAN J. H., PITOT H. C., 1970. In MUNRO, H. N. Mammolion protein metabolism, vol. 4, 388-443, Acad. Press, N. Y., London.

MALETTE L. E., EXTON J. H., PARK C. P., 1969. Effects of glucagon on amino acid transport and utilization in the perfused rat liver. J. biol. Chem., 244, 5724-5728.

Me DANIEL M. G., 1975. Acute suppression of hepatic gluconeogenesis by glucose in intact animal. Amer. J. Physiol., 229, 1569-1575.

MUNRO H. N., 1964. In MUNRO H. N., ALLISON J. B. Mammalian protein metabolism, vol. 1, 381-481. Acad. Press, N. Y., London.

NASSET E. S., JU J. S., 1961. Mixture of endogenous and exogenous protein in the alimentary canal. J. Nutr., 74, 461-465.

NASSET E. S., JU J. S., 1975. Amino acid in gut contents and blood plasma of rats as affected by dietary amino acid imbalance. J. Nutr., 105, 69-79.

PARSONS D. S., VOLMAN-MITCHELL H., 1974. The transamination of glutamate and aspartate during absorption in vitro by small intestine of chicken, guinea-pig and rat. J. Physiol., 239, 677-694.

PERET J., CHANEZ M., COTA J., MACAIRE I., 1975. Effects of quantity and quality of dietary protein and variation in certain enzyme activities on glucose metabolism in the rat. J. Nutr., 105, $1525-1534$

RERAT A., CORRING T., LAPLACE J. P., 1976. In COLE D. J. A. ef al. Protein mefabolism and nutrition, 97-138, Butterworths, London.

TEWS J. K., COLOSI N. W, HARPER A. E., 1975. Effect of amino acid meals on hepatic $\alpha$-amino isobutyrate transport and cyclic AMP. Am. J. Physiol., 228, 1606-1614.

WINDMUELLER H. G., SPAETH A. E., 1975. Metabolism of glutamine and glutamic acid during their intestinal transport in vivo. Fed. Proc., 34, 880.

YAMAMOTO H., AIKAWA T., MATSUTAKA H., OKUDA T., ISHIKAWA E., 1974. Interorganal relationship of amino acids in fed rats. Am. J. Physiol., 226, 1428-1433. 